\title{
Double-sided split-step MM-wave Fresnel lenses: design, fabrication and focal field measurements
}

\author{
V. B. Yurchenko \\ v.yurchenko@nuim.ie \\ M. Ciydem \\ mehmet.ciydem@engitek.com.t \\ M. Gradziel
}

J. A. Murphy

\section{A. Altintas}

\author{
IRE NASU, Kharkiv, Ukraine \\ Gazi University, Ankara, Turkey \\ Engitek Ltd., Ankara, Turkey \\ NUI Maynooth, Maynooth, Ireland \\ NUI Maynooth, Maynooth, Ireland \\ Bilkent University, Ankara, Turkey
}

Design, fabrication and focal field measurements of W-band Fresnel lenses to produce well-shaped Gaussian beams in the focal domain are presented. Two lenses, one of Teflon and another of Polyamide, have been compared in their performance with a double-sided Fresnel lens of different design, with full height of Fresnel steps. Experiments show that a good focusing ability of all the lenses is achieved. They produce the target beam according to the design. Yet, the lenses of split-step design are thinner, have lower insertion losses, and a greater focal depth as compared to more conventional Fresnel lenses.

[DOI: http://dx.doi.org/10.2971/jeos.2014.14007]

Keywords: Fresnel lenses, focal fields, Gaussian beam, MM-wave, THz applications

\section{INTRODUCTION}

We report on design, fabrication and focal field measurements of double-sided split-step mm-wave Fresnel lenses designed for the operation in the frequency range of $f=75-110 \mathrm{GHz}$ (W-band) and specifically optimized for the frequency $f_{0}=95 \mathrm{GHz}$. The lenses are needed for the development of light-controllable mm-wave photonic-crystal beam switches to be reported elsewhere. A pair of lenses is required for initially focusing a source beam onto the switch as a device under test (DUT) and then refocusing the exit beam for matching the pattern of a receiver horn. A view of quasioptical bench implementing this measurement setup is shown in Figure 1. The measurements are carried out using a vector network analyzer (VNA) facility operating at the Experimental Physics Department of the NUI Maynooth (Maynooth, Ireland).

A variety of lenses could, in general, be proposed for this kind of setup, starting from common smooth-wall bi-convex lenses (or planar-convex ones, often used as condensers) [1] and proceeding to Fresnel lenses and more complicated diffractive optical elements (DOE) which could be of very diverse type, e.g., imaging or non-imaging ones, digital multi-phase or multilevel zone plates, harmonic and multi-order Fresnel lenses, canonical one-sided or more complicated double-sided ones, etc [2]-[8].

For the problem at hand, however, smooth-wall or conventional Fresnel lenses are not suitable because of significant constraints arising in the experiment. The matter is that the length of quasi-optical bench is limited (less than 1.5 meters) whereas quasi-Gaussian beams of feed horns are rather wide (half-angle of the beam divergence at $f_{0}=95 \mathrm{GHz}$ is 13 degrees when following conventional definition [1] that means $88 \%$ of beam power is confined in the cone of this angle at the edge taper of $-9 \mathrm{~dB}$ ). Therefore, for the proper focusing of source beams, the lenses should have sufficient focusing power, i.e., a short focal length. In the same time, spurious signals observed at the switch have to be limited at the level of $-40 \mathrm{~dB}$ or so, depending on the kind of the structure being considered. Therefore, the lenses should be of sufficiently large diameter $\mathrm{D}$ so as to capture the incident beam down to this power level as the edge taper.

In addition, and that is of primary importance, the lenses have to produce a beam with sufficiently flat wave front on the switch structure. That means quasi-optical Fresnel lenses should be of the "imaging" kind, with minimal aberrations, so as to serve as nearly ideal transformers of divergent quasiGaussian beams of feed horns into convergent beams close to the Gaussian shape as much as possible. In the meantime, canonical Fresnel lenses being, typically, of one-sided planarconvex design are, usually, more suitable for "non-imaging" applications where significant aberrations are acceptable.

The aim of this work is, therefore, to produce mm-wave Fresnel lenses suitable for the given experiments and to verify 


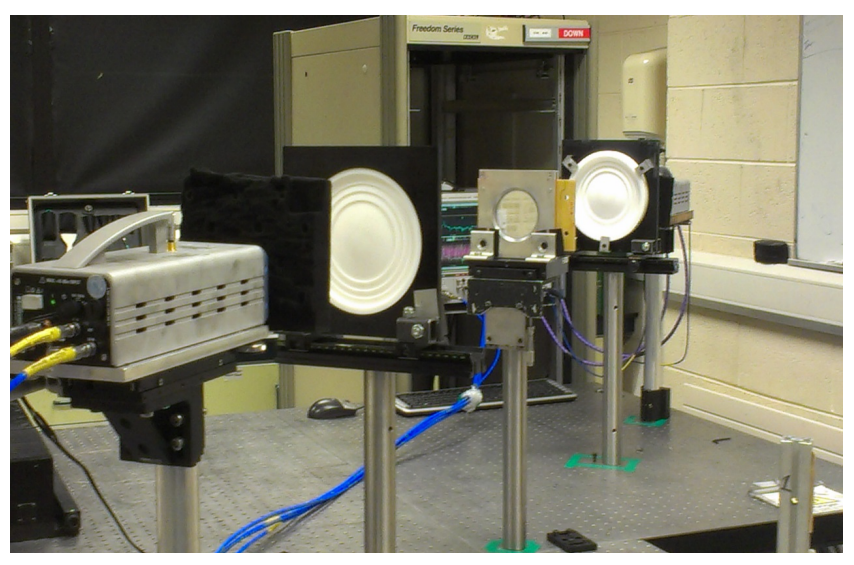

FIG. 1 Quasi-optical setup for mm-wave experiments at the NUI Maynooth, Ireland.

their performance by direct measurements of the wave field in the focal domain of these lenses.

\section{DESIGN AND FABRICATION OF LENSES}

In practice, we need lenses of clear aperture diameter $\mathrm{D}_{\mathrm{C}}=140 \mathrm{~mm}$ for capturing the beam of feed horn at the edge taper of $\mathrm{P}_{\text {edge }}=-40 \mathrm{~dB}$ when the horn aperture (of radius $\mathrm{a}=7.14 \mathrm{~mm}$ ) is at the distance $\mathrm{L}_{S 1}=125 \mathrm{~mm}$ from the front lens surface (S1). The exit beam is focused on the DUT of clear aperture $D_{0}=72 \mathrm{~mm}$ where the beam width should be $\mathrm{W}_{0}=17-24 \mathrm{~mm}$ depending on the DUT structure. The DUT is located at $\mathrm{L}_{\mathrm{S} 2}=450-435 \mathrm{~mm}$ from the rear lens surface (S2) that corresponds to the fixed distance $\mathrm{L}_{2}=\mathrm{L}_{\mathrm{S} 2}+\mathrm{H}=470 \mathrm{~mm}$ from the front surface used as a reference (an approximate Fnumber of a lens is about $\mathrm{F} / 0.7$ ). Here, $\mathrm{H}$ is the lens thickness that varies in the range of $\mathrm{H}=20-35 \mathrm{~mm}$ for our Fresnel lenses made of different materials (Teflon and Polyamide) but may be up to $\mathrm{H}=55 \mathrm{~mm}$ for non-Fresnel lenses if made of Teflon.

Because of non-paraxial character of the system and the need of obtaining a perfect beam in the focal domain, the lens should be of a special aspheric profile. Following conventional solutions, non-paraxial lenses of planar-convex type are usually employed in such cases. This is explained by relative simplicity of their design. In this case, there is only one surface of special profile that has to be defined and the profile can easily be computed when the system parameters are specified. In our case, though, this results in a bulky and heavy lens, with large incidence angles near the rim that may even approach the angles of total internal reflection at the exit surface. As a better solution, a lens of dual-aspheric profile can be used that allows one to reduce the angles of incidence and transmission, though it does not help essentially in reducing the lens thickness.

So, instead of a lens of smooth-wall design, a dual-aspheric double-sided Fresnel lens was proposed and fabricated earlier. The lens is made of Teflon, optimized for the frequency $f_{0}=100 \mathrm{GHz}$ (wavelength $\lambda_{0}=3 \mathrm{~mm}$ in free space), and has much lower thickness $(\mathrm{H}=35 \mathrm{~mm})$ as compared to lenses of a smooth-wall design $(\mathrm{H}=55 \mathrm{~mm})$. The lens has Fresnel steps on both surfaces. This makes it looking similar to $\mathrm{THz}$ lenses proposed in [8], though it has a different design. The steps on opposite surfaces in this mm-wave lens are carefully aligned in pairs, one with respect to another, along the relevant "rays" of the given beam so as to introduce a minimal wave disturbance from each pair of steps. In the meantime, each step of the conjugate pair is of the full height of modulo $2 \pi$ that means it produces a jump in the optical path in one wavelength.

Later, an improved design was proposed when two steps of each conjugate pair taken together are of the full height of modulo $2 \pi$, though each step is only a fraction of the full height. Fresnel lenses of this kind are of smaller thickness, though, possibly, of less perfect quality (but this has to be verified) as compared to either "full-step" Fresnel lenses (whether of one-sided or double-sided profile) or classical smoothsurface lenses (non-Fresnel ones). In the same time, in any of these lenses, smooth surface zones between the steps are computed as special aspheric surfaces, which provide smooth transformation of the incident Gaussian beam into the required exit beam, also of Gaussian type, though convergent towards the DUT.

In this work, we report on fabrication of two sets of doublesided split-step dual-aspheric Fresnel lenses optimized for the operation frequency of $f_{0}=95 \mathrm{GHz}$. One set of lenses (those shown in Figure 1) are made of Teflon (lenses \#1), having thickness $\mathrm{H}=30 \mathrm{~mm}$, and designed by assuming Teflon permittivity $\epsilon=2.00$. The other lenses (\#2) are made of Polyamide, being of thickness $\mathrm{H}=20 \mathrm{~mm}$, and designed assuming $\epsilon=2.80$ as found for the given material in our earlier measurements [9]. The lenses are fabricated by using a CNC technique following the design described above.

Split-step Fresnel lenses of both materials were designed to provide the same beam on the DUT in the focal plane, with the beam width $W_{0}=24 \mathrm{~mm}$ and the edge taper of $-20 \mathrm{~dB}$ at the DUT aperture $\mathrm{D}_{0}=72 \mathrm{~mm}$. These parameters were chosen for reducing the beam convergence and improving the wavefront flatness at the DUT that should improve the functionality of switch structures. Contrary to this, full-step doublesided Fresnel lenses of an older design, also made of Teflon, were fabricated earlier (lenses \#3) to provide a smaller beam width of about $\mathrm{W}_{0}=17 \mathrm{~mm}$ on the DUT for reducing the edge taper down to $-40 \mathrm{~dB}$.

\section{FOCAL FIELD MEASUREMENTS}

For detailed characterization of these lenses, we carried out measurements of their field distributions in the focal domain (both the field power and the phase) using the VNA scanner facility at the NUI Maynooth (Maynooth, Ireland). The measurements were made in the frequency band of $75-110 \mathrm{GHz}$ when scanning through the frequency with the increment of $5 \mathrm{GHz}$. A special care was taken to ensure sufficiently perfect alignment of quasi-optical system, which is a very crucial issue in this experiment.

The measurement results obtained for three kinds of Fresnel lenses are presented in Figures 2 to 4 . The plots show the field power (presented in decibels and in the relative units) and the phase (in degrees) spatial distributions in the horizontal cut of 


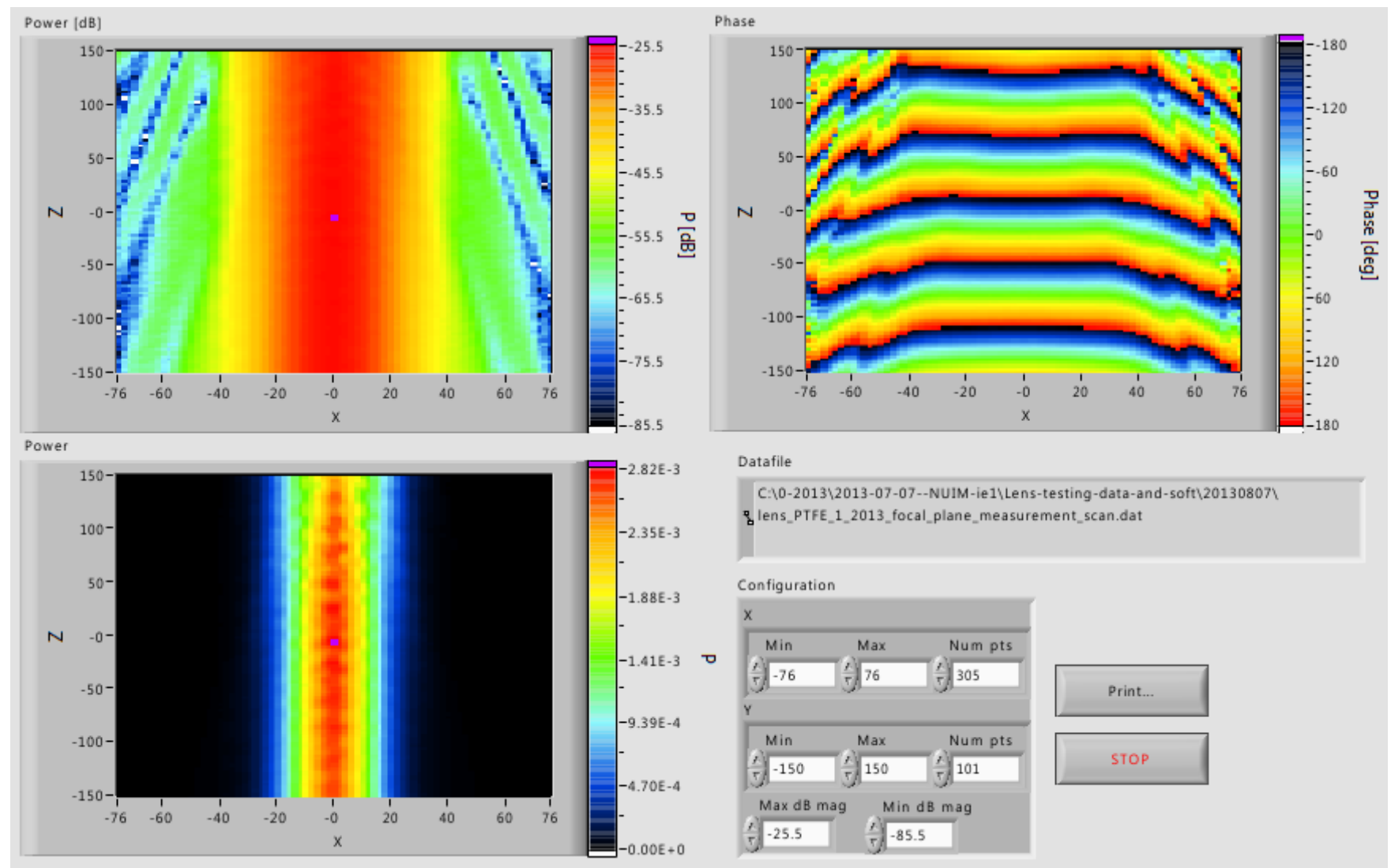

FIG. 2 Focal field power and phase distributions as measured for the split-step dual-aspheric double-sided Fresnel lens of new design made of Teflon (lens \# $1, \mathrm{H}=30 \mathrm{~mm}$ ).
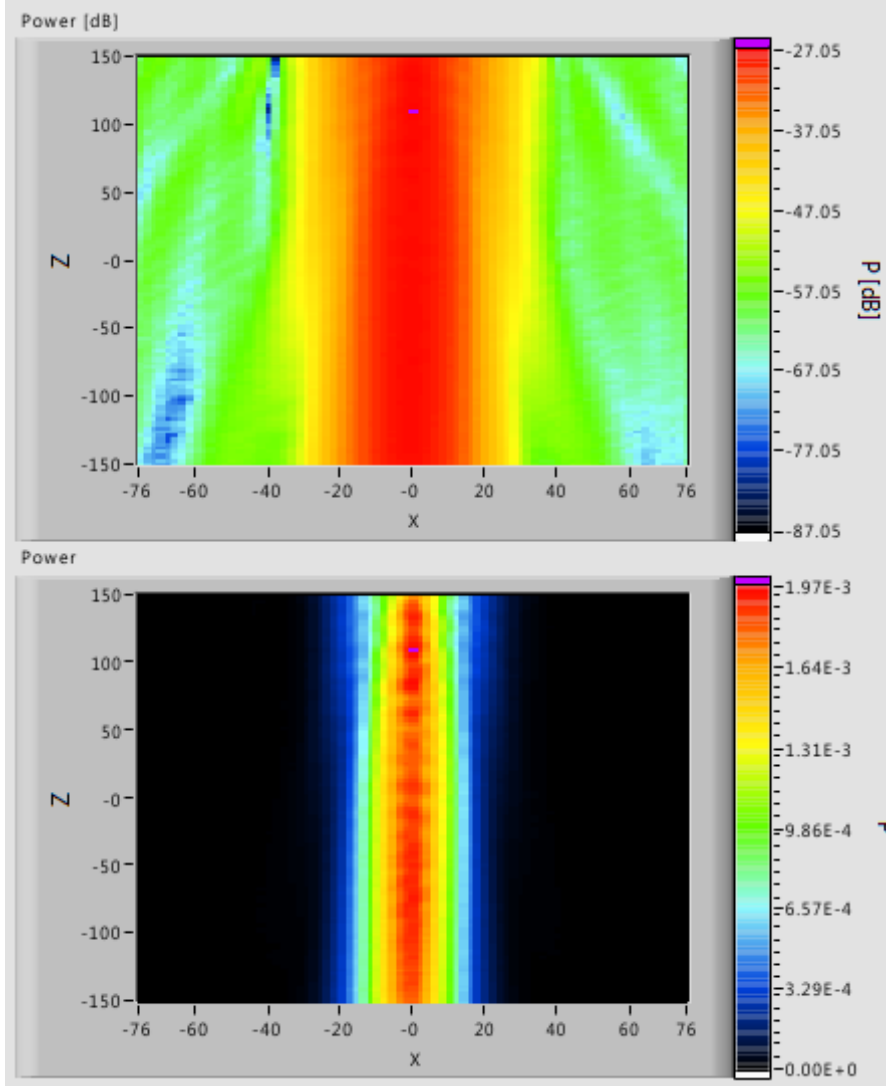

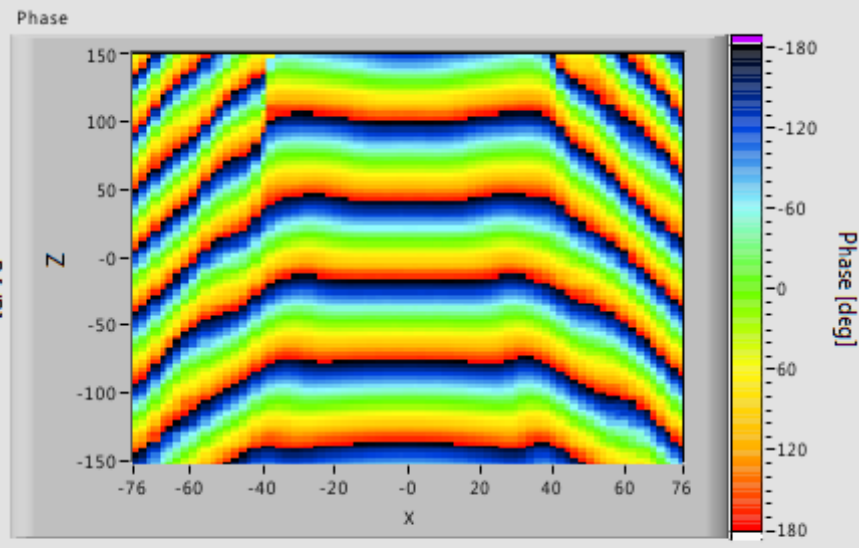

Datafile

C:\0-2013\2013-07-07--NU1M-ie1\Lens-testing-data-and-soft $20130808 \backslash$ g. Iens_PA_1_2013_focal_plane_measurement_scan.dat

Configuration

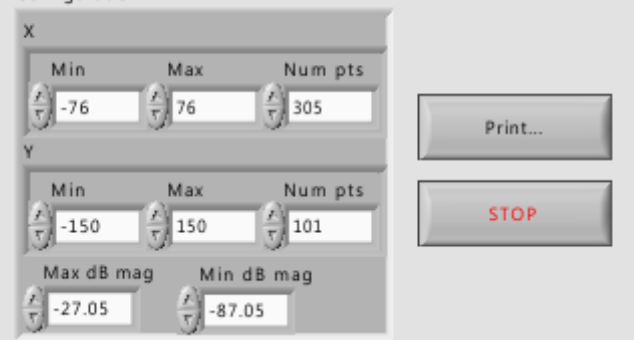

FIG. 3 Focal field power and phase distributions as measured for the split-step dual-aspheric double-sided Fresnel lens of new design made of Polyamide (lens \#2, $\mathrm{H}=20 \mathrm{~mm}$ ). 


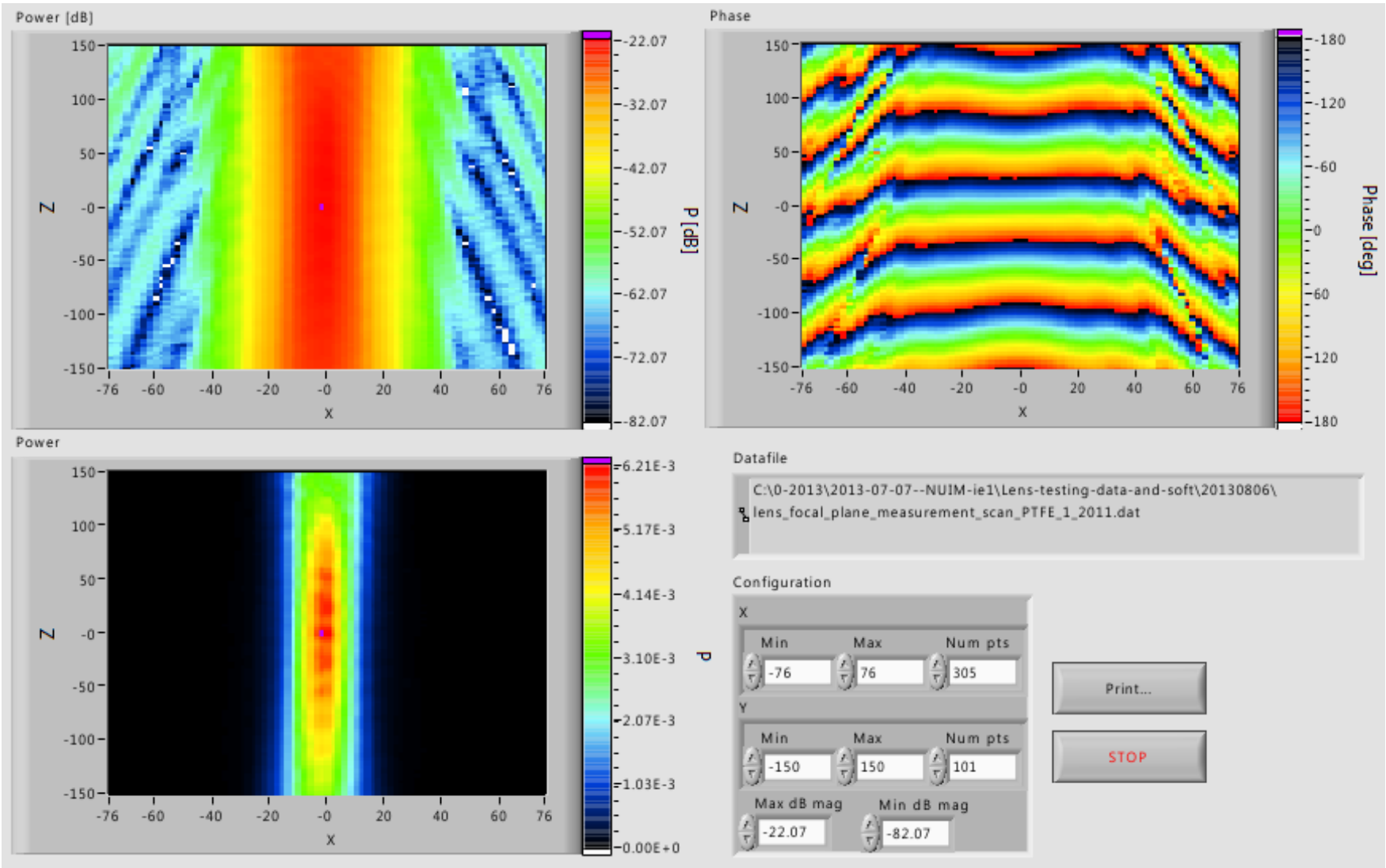

FIG. 4 Focal field power and phase distributions as measured for the full-step dual-aspheric double-sided Fresnel lens of old design made of Teflon (lens \#3, $\mathrm{H}=35 \mathrm{~mm}$ ).

the focal domain behind the lens (the $\mathrm{XZ}$ plane, where $\mathrm{Z}$ is the axial coordinate with the origin $Z=0$ placed at the expected focus position and $Z>0$ pointing away from the source horn). The plots are built for all three lenses at the same operation frequency $f_{0}=95 \mathrm{GHz}$ (notice, the phase plots are shown with aliasing effects because of too small wavelength for a clear presentation of these plots in a more detailed manner).

Focal field measurements confirmed a good focusing ability of all three kinds of lenses considered. Quantitative analysis shows that the target beam width values $W_{0}=17 \mathrm{~mm}$ and $\mathrm{W}_{0}=24 \mathrm{~mm}$ are achieved with relevant lenses as expected.

The best results are, naturally, obtained with lenses made of Teflon as the material of choice for mm-wave applications. Yet, a thinner lens of Polyamide has also shown a good performance despite greater losses in this material. The focal field of this lens, though less structured with troughs and sidelobes outside the main beam, is well shaped in the main area and, more so, shows a significant focal depth that is a useful quality in many applications.

The quality of having an increased focal depth seems to be a characteristic feature of split-step Fresnel lenses (\#1 and \#2) whereas the full-step lens (\#3) has a shorter focal depth (this can be seen by comparing the green zones in the relative power plots where the green color indicates the halfmagnitude power level). The effect, though, may be merely a result of a smaller thickness of a dual-aspheric lens when a split-step Fresnel profile is implemented.
All the lenses show some standing waves in the focal area, which is unavoidable in any system. Yet, again, a lens of Polyamide shows a bit peculiar property of having the "focal point" as if split in two points along the beam axis, which are located at about $100 \mathrm{~mm}$ one before and another after the main "focal point" expected at $Z=0 \mathrm{~mm}$. This is in line with the fact of extended focal depth obtained with split-step Fresnel lenses.

\section{CONCLUSIONS}

We made focal field measurements of double-sided split-step dual-aspheric mm-wave Fresnel lenses designed for producing well-shaped Gaussian beams in the focal domain. Two lenses, one of Teflon and another of Polyamide, were compared in their performance with a double-sided Fresnel lens of different design, with full height of Fresnel steps.

We confirm a good focusing ability of all the lenses. They produced the target beam width according to the design. Yet, the lenses of split-step design are thinner, have lower insertion losses, and a greater focal depth as compared to more conventional Fresnel lenses.

MM-wave lenses made of lossy materials could serve as a good model for the analysis of lenses for $\mathrm{THz}$ applications where losses are always significant and unavoidable. 


\section{ACKN OWLEDGEMENTS}

Efforts have been sponsored by the Air Force Office of Scientific Research, Air Force Material Command, USAF, under grant number FA8655-12-1-2073. The U.S Government is authorized to reproduce and distribute reprints for Governmental purpose notwithstanding any copyright notation thereon.

Publication has partially been supported by The Scientific and Technological Research Council of Turkey (TUBITAK) within Science Fellowship and Grant Program "2221 Fellowships for Visiting Scientists and Scientists on Sabbatical Leave". V. Y. and M. C. are grateful to M. T. Aydemir for his interest in this research.

\section{References}

[1] E. Hecht, Optics (Addison-Wesley, Reading, 1998).

[2] J. Turunen, and F. Wyrowski, Diffractive optics for industrial and commercial applications (Akademie Verlag and J. Wiley, Berlin, 1998).
[3] B. Kress, and P. Meyrueis, Digital diffractive optics: An introduction to planar diffractive optics and related technology (John Wiley and Sons, Chicester, 2000).

[4] R. Leutz, and A. Suzuki, Nonimaging Fresnel lenses: Design and performance of solar concentrators (Springer, New York, 2001).

[5] I. V. Minin, and 0. V. Minin, Diffractional optics of millimetre waves (Institute of Physics, Bristol, 2004).

[6] D. W. Sweeney, and G. E. Sommargren, "Harmonic diffractive lenses," Appl. Optics 34, 2469-2475 (1995).

[7] D. Faklis, and G. M. Morris, "Spectral properties of multiorder diffractive lenses," Appl. Optics 34, 2462-2468 (1995).

[8] M. Sypek, M. Makowski, E. Hérault, A. Siemion, A. Siemion, J. Suszek, F. Garet, et al., "Highly efficient broadband double-sided Fresnel lens for THz range," Opt. Lett. 37, 2214-2216 (2012).

[9] V. B. Yurchenko, A. Altintas, M. Ciydem, and S. Koc, "Experimental conditions for the excitation of thin disk whispering-gallery-mode resonators," Prog. Electromagn. Res. C 43, 29-40 (2013). 